\title{
Age- and species-specific duration of infection in asymptomatic malaria infections in Papua New Guinea
}

\author{
M. C. BRUCE ${ }^{1 *}$, C. A. DONNELLY ${ }^{1}$, M. PACKER ${ }^{2}$, M. LAGOG ${ }^{2}$, N. GIBSON ${ }^{2}$, \\ A. NARARA ${ }^{2}$, D. WALLIKER ${ }^{3}$, M. P. ALPERS ${ }^{2}$ and K. P. DAY ${ }^{1}$ \\ ${ }^{1}$ Wellcome Trust Centre for the Epidemiology of Infectious Disease, Department of Zoology, University of Oxford, \\ Oxford OX1 $3 F Y$ \\ ${ }^{2}$ Papua New Guinea Institute of Medical Research, P.O. Box 378, Madang, Papua New Guinea \\ ${ }^{3}$ Institute of Cell, Animal and Population Biology, University of Edinburgh, King's Buildings, Edinburgh EH9 $37 N$
}

(Received 9 November 1999; revised 16 March 2000; accepted 16 March 2000)

SU M M A R Y

The burden and duration of asymptomatic malaria infections were measured in residents of the malaria endemic village of Gonoa, Madang Province, Papua New Guinea. Plasmodium falciparum, P. vivax and P. malariae infections in people aged 4 years to adulthood were compared. Frequent sampling at 3 -day intervals for up to 61 days allowed assessment of individual episodes of infection. Statistical assessment of $P$. falciparum detection revealed a periodicity consistent with synchronous replication of this species over periods up to 27 days. The duration of $P$. falciparum episodes was longer across all age groups than that of $P$. vivax and $P$. malariae. A trend for decreasing duration with age was also noted in data from each species. This was most prominent in $P$. falciparum infections: median duration in 4-year-olds was $>48$ days compared with a median between 9 and 15 days in older children and adults. The results are consistent with the slow acquisition of immunity to antigenically diverse Plasmodium populations and suggest a faster rate of acquisition to $P$. vivax and P. malariae than to P. falciparum.

Key words: Plasmodium falciparum, Plasmodium vivax, Plasmodium malariae, asymptomatic, age-specific duration, species-specific duration.

\section{INTRODUCTION}

The majority of malaria infections in individuals living in endemic regions are asymptomatic. Even young children, who bear the highest burden of disease carry asymptomatic infections for most of the time (Greenwood, 1987; Greenwood et al. 1987). Malaria prevalence is estimated from samples of such asymptomatic infections and prevalence studies show that for all 4 human malaria species, peak infection rates occur in children and decline with age. This decline of prevalence with age is widely held to be a result of acquired immunity which requires multiple infections to develop.

Most surveys of the burden of malaria infection use cross-sectional sampling. However, this provides a rather crude measure because prevalence is a result of several factors including the incidence of new infections, the rate of relapse of latent infections and the rate of recovery from infection (Molineaux, 1988). Acquired immunity can affect the dynamics of infection by reducing the duration of infection and by lowering the incidence of infections which result in detectable parasitaemia. In order to understand

* Corresponding author: Wellcome Trust Centre for the Epidemiology of Infectious Disease, Department of Zoology, University of Oxford, Oxford OX1 3FY. Tel: +01865 271247. Fax: +01865 281245.

E-mail: marianbruce@hotmail.com the contribution of such factors to the dynamics of infection, parasites present within individual patients must be tracked over time. The density of parasites and the species present, as well as individual episodes of a species must be determined. Few longitudinal studies of this type have been done and these have either concentrated on a single age group (Hill, Cambournac \& Simoes, 1943; Bruce-Chwatt, 1963 ; Gilles, 1993) or do not give sufficiently detailed data for re-analysis (Earle et al. 1939; Miller, 1958).

In this paper, we describe longitudinal surveys of malaria infections in individuals living in a highly malaria-endemic region of Papua New Guinea where mixed infections of $P$. falciparum, $P$. vivax and $P$. malariae are common. We examine parasites among the inhabitants of a single village, aged from 4 years to adulthood to determine whether there are differences in the dynamics and duration of each species across and within different age-groups. In the following paper we analyse the infections in more detail to examine the dynamics of individual genotypes of $P$. falciparum and $P$. vivax in children (Bruce et al. $2000 b)$.

MATERIALS AND METHODS

Study area

All participants were residents of the village of Gonoa, Madang Province, Papua New Guinea (Fig. 

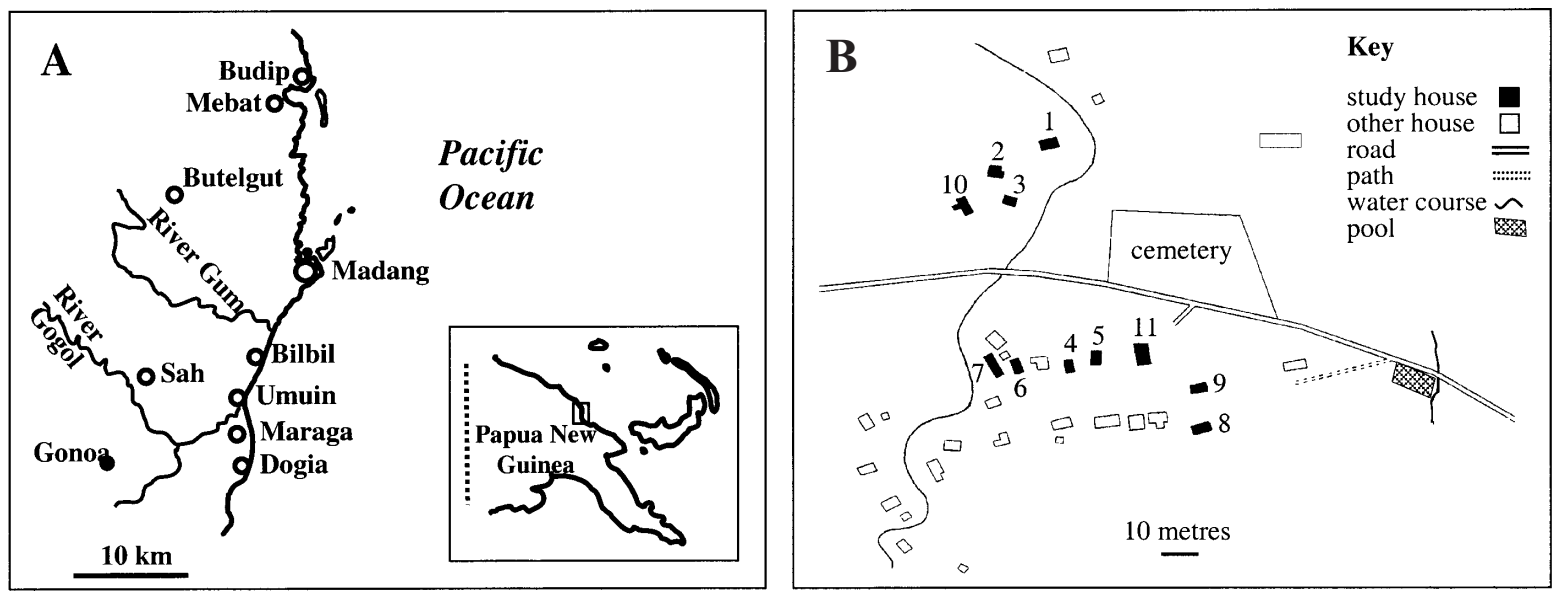

Fig. 1. The village of Gonoa (A) geographical location and (B) map of the village showing the position of study houses. Inhabitants of houses 4 and 5 , and 6 and 7 were two family units each residing in two dwellings.

$1 \mathrm{~A})$. The village lies in an area of secondary forest, $4 \mathrm{~km}$ from the Gogol River and has approximately 200 residents. The majority of inhabitants were selfemployed farmers. A few have paid employment with a local logging firm. Year round transmission of all 4 species of Plasmodium which infect humans (Plasmodium falciparum, P. vivax, P. malariae and $P$. ovale), occurs in this region (Cattani et al. 1986). The study was carried out from 28 June to 27 August 1992, during the drier season of the year, which lasts from June to September. The mean number of infectious bites per person per day in this region has been estimated at 0.86 (Burkot et al. 1987) but during the drier season, transmission is reduced to about $1 / 3$ of that during the wet season (Burkot et al. 1988). Members of the Anopheles punctulatis Dönitz complex, An. funestus, An. punctulatis and An. koliensis are the vectors (Burkot et al. 1988). The village has an aid post at which free malaria treatment is available. Previous studies in the area indicate that most treatment is sought through health centres but that purchase of anti-malarial drugs and selftreatment does occur (Cattani et al. 1986; Cox et al. 1994).

\section{Study population and sampling protocol}

Study houses were selected on the basis of compliance with the sampling protocol. All consenting inhabitants of the 11 study houses (Fig. 1B), aged 4 years or greater (and 1 child aged 3 years, included at the request of the parents), were enrolled into the study $(n=70)$ following detailed explanation of the study protocol in the local Pidgin language. Verbal consent for children was given by parents or guardians. Eight children under 4 years and 1 adult were not enrolled. For the purposes of comparison with previous malaria surveys (Cattani et al. 1986), individuals were categorized using the following age groups: 3-4 years, 5-9 years, 10-14 years and adults, who were individuals greater than 14 years. Bed net use was $100 \%$ although nets were not treated with insecticide and many were damaged.

Samples of peripheral blood were collected via fingerpricks from individuals every third day for a period of 61 days as previously reported (Bruce et al. $2000 a$ ). Samples from adults were taken only for the first 28 days (10 time-points). Thick and thin blood smears were prepared for microscopy from fingerpricks. At the same time, blood was also collected for use in genetic analyses of Plasmodium spp. (Bruce $e t$ al. 2000 b). Sampling ceased for 2 time-points, days 45 and 48 , due to the death of a non-enrolled, elderly villager. Only individuals for whom $>50 \%$ of samples were available (out of 19 possible samples for children and 10 for adults), were used in analyses. Clinical disease was monitored by self-reporting at 3-day intervals. Ethical clearance was granted for longitudinal surveillance in the absence of treatment by the Medical Research Advisory Committee of Papua New Guinea, who advise non-treatment of asymptomatic malaria infections to allow natural, protective immunity to develop.

\section{Microscopy}

Blood smears were air dried and thin smears were methanol fixed. Both thick and thin smears were stained in $4 \%$ Giemsa solution in sodium phosphate buffer, $\mathrm{pH} 7 \cdot 2$, for $45 \mathrm{~min}$. Species identification and enumeration of parasite density was obtained from examination of both thick and thin smears. $P$. falciparum asexual and gametocyte stages were counted separately but counts were combined for all analyses to allow direct comparison with other species. All stages of the other species, $P$. vivax, $P$. malariae and $P$. ovale were counted together. Parasite density for all species was taken as the sum of asexual stages and gametocytes. Parasite counts were made per 200 leukocytes then converted to counts per $\mu 1$ of blood assuming a standard leukocyte count of 8000 per $\mu \mathrm{l}$. Duplicate readings were made for $20 \%$ of 
smears. Greater than $75 \%$ concordance in results was observed and therefore initial results were accepted.

\section{Defining species-specific episodes of infection}

Episodes of infection were defined by consecutive smear positive time-points for $P$. vivax and $P$. malariae. To adjust for periodicity in the detection of $P$. falciparum parasitaemia (see Results section), consecutive smear-positive samples or those smear positive at 6-day intervals were used to define episodes of this species. The start of an episode was taken as the mid-point between the first positive sample and the previous negative sample. Similarly, an episode's end was defined as the mid-point between the last positive sample and the next negative one. Where episodes overlapped the beginning or end of the study period, these were used as end-points. A maximum of 2 consecutive missing data points was tolerated. When this was exceeded, episodes were assumed to have terminated at the mid-point between the last positive sample and the first missing data point. All duration estimates were rounded to multiples of 3 days.

\section{Statistical analysis}

Periodicity in the detection of each Plasmodium species within individuals was analysed using a paired method. The probability of detection of a second smear-positive sample, conditional on the first in the pair being positive, was calculated for all possible pairs of samples from all individuals, at intervals between 3 and 60 days. Data from each interval were then pooled across individuals by age group. The $95 \%$ confidence intervals were estimated as $\pm 1.96 \times 2$ (standard error). Exact $95 \%$ confidence intervals were calculated for probability estimates close to zero and for those time-intervals with few data pairs.

The Kaplan-Meier or survival estimator was used to analyse the duration of episodes of infection defined by microscopy. Data from individuals in each age group were pooled. Separate analysis of data from each person was precluded due to the paucity of data from each individual. Estimates were considered censored if episodes overlapped the beginning or endpoint of the study period. The distributions of estimates from different species and age groups were compared using a $\chi^{2}$ test. Unadjusted $P$-values are reported, so as to allow access to raw test results. It must be borne in mind that comparisons are subject to the constraints of multiple testing.

RESULTS

Seventy individuals aged 4 to $>50$ years were enrolled into the study, constituting $88 \%$ of the members of 9 family units, living in 11 houses in the village of Gonoa, Papua New Guinea (Fig. 1). Blood smears were taken every third day for a period of 29 days (a total of 10 time-points). Sampling was continued in children, aged 4-14 years, for a further 32-day period (a further 11 time-points).

All individuals were asymptomatic by selfreporting, except for 1 child aged 6, who was treated for malaria symptoms and subsequently removed from the analysis. Data from people with greater than $50 \%$ compliance with sampling protocols were age-stratified according to categories formerly used in large-scale malaria surveys in Madang, to allow direct comparison with previous data (Cattani et al. 1986). The numbers of individuals in each age group were as follows: 4 years $n=5,5-9$ years $n=13$, $10-14$ years $n=16,>14$ years (adults) $n=24$.

\section{Prevalence of Plasmodium species}

The presence and density of Plasmodium species at each time-point was assessed by microscopy. All 4 species were detected in samples in this study. Parasites were detected in all but 1 person over the duration of the study. The numbers of positive blood smears for each species are given for all individuals in a summary table which can be accessed at http://www.ceid.ox.ac.uk/download/. A comparison of the prevalence of infection in each age group was made between mean daily prevalence (point prevalence) and cumulative prevalence observed over days $0-27$ of the study (period prevalence). Point prevalence for all species and age groups ranged from 0 to 0.5 (Fig. $2 \mathrm{~A}$ ).

A large increase can be seen across all age groups when mean daily values are compared to longitudinal data from the same period (period prevalence, Fig. $2 \mathrm{~B})$. The factor by which the prevalence estimate was elevated between point and period prevalence increases with age and was greatest for $P$. malariae infections in adults (Table 1).

\section{Parasite density}

Parasite density in children (aged 4-14 years) ranged from 40 to 11520 parasites $/ \mu$ l for $P$. falciparum, from 40 to 7600 parasites $/ \mu$ l for $P$. vivax and from 40 to 1640 parasites $/ \mu$ for $P$. malariae. $P$. ovale was found in a single sample from 1 child at a density of 5080 parasites $/ \mu 1$. Density of all species was much reduced in adults (aged $>14$ years) compared to children. Ranges in density were from 40 to 4080 parasites $/ \mu 1$ for $P$. falciparum, from 40 to 320 parasites $/ \mu 1$ for $P$. vivax and from 80 to 400 parasites $/ \mu 1$ in $P$. malariae infections. $P$. ovale was not detected in adults. Gametocytes of $P$. falciparum were detected in only 10 samples from 5 children and 2 adults. Density was low in all cases, ranging from 40 to 80 gametocytes $/ \mu 1$. Gametocytes of other species were not counted separately. Geo- 

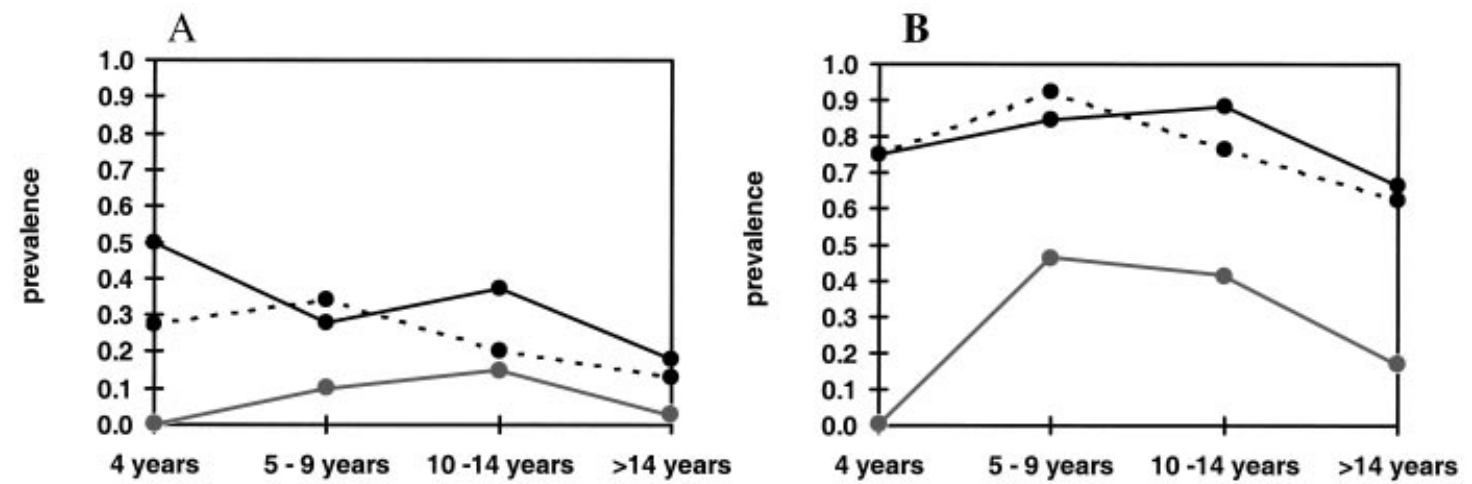

Fig. 2. Age-specific prevalence of infection estimated from (A) the mean point prevalence over days 0-27 of the study, and (B) period prevalence calculated from longitudinal data over the same time-interval. Data for Plasmodium falciparum is shown with a solid black line, $P$. vivax with a broken line and $P$. malariae with a grey line. Only individuals from whom $>50 \%$ of samples ( 5 or more samples out of a possible 10) were obtained during days $0-27$ were used to calculate these values. Children 59, 60 and 61 (see summary table at http://www.ceid.ox.ac.uk/ download/), were used here but are not used in duration of infection analyses due to having fewer than $50 \%$ of samples over days 0-60 of the study. Children 4, 11 and 29 who do contribute data to duration analyses are not used here as the number of samples obtained over days $0-27$, was less than $50 \%$. The number of individuals used in this comparative prevalence analysis are as follows: 4 years, $n=4$; $5-9$ years, $n=13$; $10-14$ years, $n=17$; $>14$ years, $n=$ 24. The number of samples positive during days $0-27$ is shown for each individual in the summary table at http://www.ceid.ox.ac.uk/download/.

Table 1. The factor by which prevalence increases with age when point prevalence (mean daily prevalence over days $0-27$ ) is compared to period prevalence (cumulative prevalence over the same 0-27 day period)

\begin{tabular}{lllll}
\hline \hline & & \multicolumn{3}{c}{$10-14$} \\
& 4 years & 5-9 years & years & > 14 years \\
\hline P. falciparum & 1.5 & 3.0 & $2 \cdot 4$ & $3 \cdot 7$ \\
P. vivax & $2 \cdot 7$ & $2 \cdot 7$ & $3 \cdot 8$ & 4.9 \\
P. malariae & - & $4 \cdot 7$ & $2 \cdot 8$ & $6 \cdot 1$ \\
\hline \hline
\end{tabular}

metric mean density of $P$. falciparum and $P$. vivax declined with age (Table 2). Values for P. malariae were similar between 10 and 14-year-olds and adults, whilst they were slightly elevated in the 5 to 9 -year- olds. Differences in means cannot be tested straightforwardly for significance because the data consist of multiple samples from each individual.

\section{Periodicity in the detection of parasitaemia}

To investigate evidence for periodicity of detection of each species, paired analyses of samples from each individual were carried out. The conditional probability that the second sample in any pair was smearpositive following a smear-positive result in the first sample was calculated from all possible pairs of samples. The analysis was carried out separately for P. falciparum, P. vivax and P. malariae. Data from children of all ages were pooled. Adult data were analysed independently.

The conditional probability of detection of $P$. falciparum infection in children demonstrated

Table 2. Geometric mean parasite density (parasites/ $\mu$ l of blood) in microscopy positive samples, by age group and species

(Total density is the sum of density of all species of parasite present in any single smear; $n$ is the number of smears positive for each species from which values are calculated.)

\begin{tabular}{lcccc}
\hline \hline & 4 years & $5-9$ years & $10-14$ years & $>14$ years \\
\hline$P$. falciparum & 685 & 342 & 321 & 193 \\
$n$ & 37 & 55 & 82 & 36 \\
$P$. vivax & 536 & 193 & 133 & 80 \\
$n$ & 23 & 55 & 57 & 26 \\
$P$. malariae & - & 231 & 103 & 165 \\
$n$ & 0 & 13 & 34 & 6 \\
Total density & 761 & 312 & 230 & 61 \\
$n$ & 50 & 107 & 151 & \\
\hline \hline
\end{tabular}



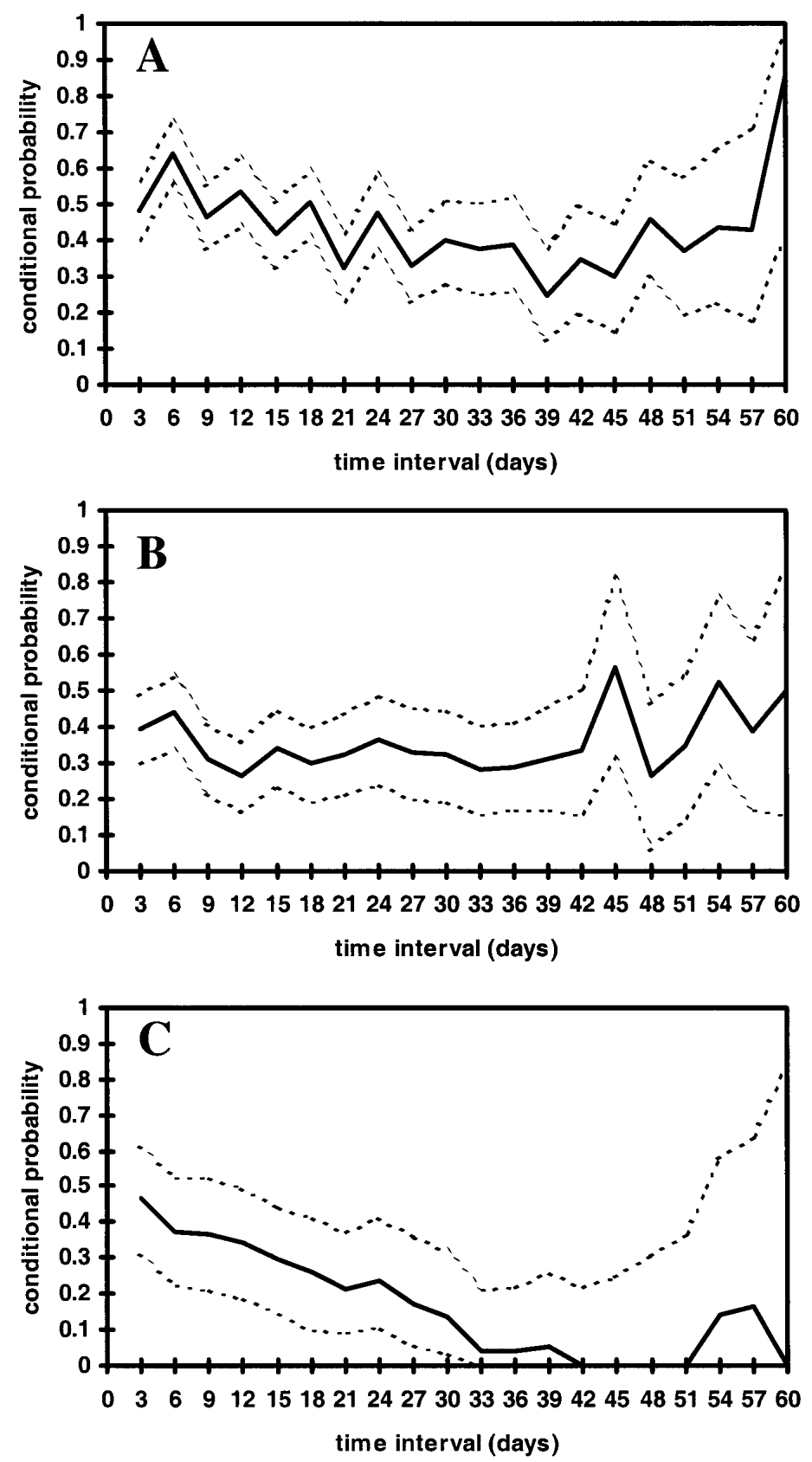

Fig. 3. Conditional probability of detection of Plasmodium species in pairs of samples from individual children aged 4-14 years, taken at 3-60 day intervals for (A) P. falciparum, (B) P. vivax and (C) P. malariae. Probability is conditional on detection of the same species in the chronologically first sample in the pair. The numbers of children with each species, used in this analysis, are as follows: $P$. falciparum $n=29, P$. vivax $n=31, P$. malariae $n=16$.

periodicity. The probability of infection was higher at $6,12,18$ and 24-day intervals than at 3,9,15, 21 and 27 days (Fig. $3 \mathrm{~A}$ ). The confidence intervals widen with increasing time-interval as the number of pairs of samples analysed decreases. Conditional probability plots for $P$. vivax and $P$. malariae in children showed no evidence of periodicity (Fig. $3 \mathrm{~B}$ and $\mathrm{C}$, respectively).

The decline in the conditional probability of infection with $P$. malariae is due to the lower prevalence of this infection compared to the other species. Most children infected with this species have only a single episode (see summary table at http://www.ceid.ox.ac.uk/download) and so probability declines with increasing time-interval. Analysis of data from adults revealed no evidence of periodicity in any species due to the paucity of smear positive samples (data not shown).

\section{Dynamics of episodes of infection}

Infection with each species of parasite followed a pattern of intermittent bursts of parasitaemia. Each burst is referred to here as an 'episode' of infection. Episodes most commonly showed increasing and then declining density. As discussed above, $P$. 

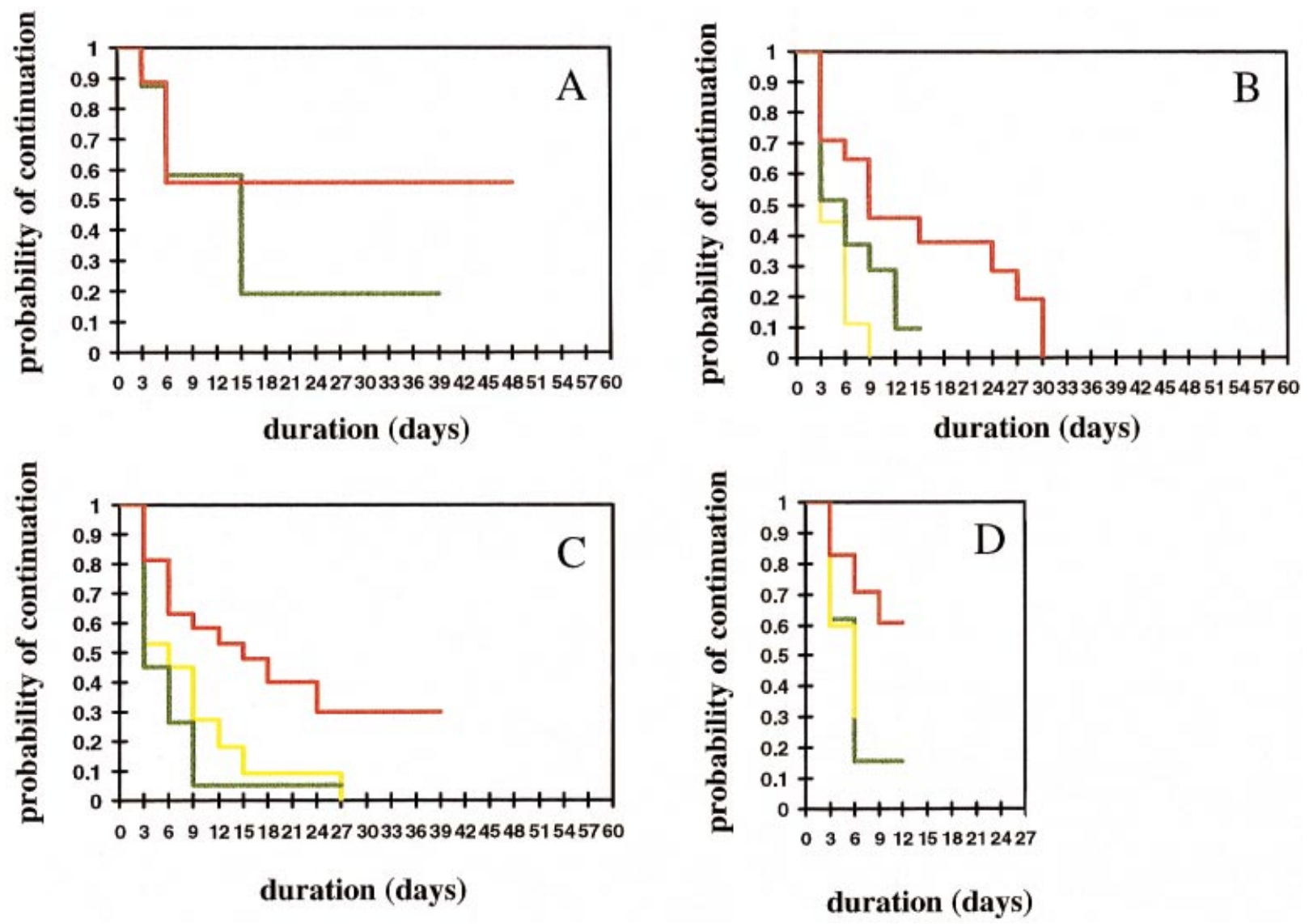

Fig. 4. Kaplan-Meier survival plots of duration of episodes of infection in children aged (A) 4 years, (B) 5-9 years, (C) 10-14 years and (D) adults (>14 years) with Plasmodium falciparum (red), P. vivax (green) and P. malariae (yellow).

falciparum parasites were detected at 6-day intervals in many individuals and these types of episodes typically followed similar dynamics. The durations of episodes were estimated and compared amongst different species of parasites and across age groups of human hosts.

The duration of episodes was quantified by measuring the number of consecutive time-points at which each parasite species was detected within individuals. The periodicity in the detection of $P$. falciparum infection (Fig. 3 A) was taken into consideration when assessing duration of episodes, to avoid underestimates for this species and to allow direct comparison with estimates for $P$. vivax and $P$. malariae. A strict set of rules for the definition of the beginning and end-points of episodes with regard to missing data-points were devised and used universally. The number of episodes of each species defined per individual is given in the summary table at http://www.ceid.ox.ac.uk/download/. The effect of combining $P$. falciparum asexual and gametocyte counts was negligible as gametocytes were found only in 10 samples from 7 individuals and in the majority of samples these were present along with asexual parasites.

Episodes of infection of all species were variable in length both between and within individuals. Esti- mates of the duration of infection in each age group and for each species are shown as Kaplan-Meier plots (Fig. 4). These plots show the probability of the continuation of an episode with increasing time. From the Kaplan-Meier plots a trend of decreasing episode duration with age is apparent in all species.

For $P$. falciparum, the difference in duration was greatest between the 4-year-olds compared to older individuals. The median duration estimate was greater than 48 days in 4-year-olds, whilst for children aged 5-9 and 10-14 years it was 9 and 15 days, respectively (Table 3 ). In adults, the median was similar to that in older children (12 days) but the maximum observed duration was less than for children. Despite these trends in the distribution of $P$. falciparum duration, estimates were not significantly different between age groups $\left(\chi^{2}=2 \cdot 6,3\right.$ D.F., $P=0 \cdot 461)$.

The distribution of duration estimates for $P$. vivax and $P$. malariae were not significantly different between age groups ( $P$. vivax, $\chi^{2}=5 \cdot 5,3$ D.F., $P=$ $0 \cdot 14, P$. malariae $\chi^{2}=2 \cdot 7,2$ D.F., $\left.P=0 \cdot 261\right)$. However, a trend of decreasing duration with age was also evident in these species. $P$. vivax infections were longest in 4-year-olds but the difference between the age groups was less marked than for $P$. falciparum (Table 3). Median values for $P$. vivax were similar 
Table 3. Median duration (in days) of episodes of infection by age group and species

(Median duration is obtained from Kaplan-Meier plots (Fig. 4); $95 \%$ confidence intervals are shown in parentheses.)

\begin{tabular}{lllll}
\hline \hline & 4 years & 5-9 years & 10-14 years & $>$ 14 years \\
\hline P. falciparum & $>48$ & 9 & 15 & 12 \\
P. vivax & $(3->48)$ & $(3-27)$ & $(6->39)$ & $(6->12)$ \\
P. malariae & 15 & 6 & 3 & 6 \\
& $(3->39)$ & $(3-9)$ & $(3-6)$ & $(3-6)$ \\
& & 3 & 6 & 6 \\
& & $(3-6)$ & $(3-12)$ & $(3->6)$ \\
\hline \hline
\end{tabular}

for older age groups (3-6 days) but, as with $P$. falciparum, the range of observed values was less than in the adult group. Median values for the duration of $P$. malariae infections were similar in all 3 age groups in which this parasite was detected (3-6 days) but the range was again more limited in adults (Table 3 ).

When data for each species were compared across age groups, a significant difference between the duration of episodes with different species was observed. P. falciparum episodes lasted longer than those of $P$. vivax or $P$. malariae in all age groups (combined age groups 4-14 years, $\chi^{2}=23 \cdot 9,2$ D.F., $P<0.001$ and age group $>14$ years, $\chi^{2}=6 \cdot 2,2$ D.F., $P=0 \cdot 044)$.

\section{DISCUSSION}

We have used longitudinal microscopy data from blood smears collected at very frequent intervals to compare the dynamics of infection of 3 malaria species $P$. falciparum, $P$. vivax and $P$. malariae among inhabitants of a single village in Papua New Guinea. We show that high levels of super-infection i.e. with more than 1 species per patient, are common in this community. The duration of episodes of infection of each species varies considerably both within and between individuals. Infections often last for several weeks in young children but generally for only a few days in adults. This variation is probably due to immunity acquired with age, resulting from multiple infections.

The decline of parasite density with age, observed in $P$. falciparum and $P$. vivax, is characteristic of both symptomatic and asymptomatic infection in endemic regions (Cox et al. 1994; Smith et al. 1995; Rogier, Commenges \& Trape, 1996). Geometric mean values are consistent with asymptomatic values found in a large-scale cross-sectional malaria survey in this region (Cox et al. 1994). The similarity in $P$. malariae density between age groups may be a result of small sample numbers, and differences between age groups cannot be excluded. The low density of
P. malariae is characteristic of this species, which does not usually reach levels of other species in either asymptomatic or symptomatic infections.

In all age groups, $P$. vivax density was lower than $P$. falciparum, consistent with previous studies in this region, which also show reduced density in both asymptomatic and symptomatic infections with $P$. vivax (Cox et al. 1994). Although fever was not reported in the study population, association of some of the higher parasitaemias in the younger children with an increase in temperature cannot be discounted, as this was not measured. Surveillance of fever in this region showed that $P$. falciparum parasitaemia above 5000 parasites / $\mu$ l was more likely to be associated with a febrile illness but that symptoms often go unreported (Cox et al. 1994).

The likely cause of the periodicity observed in $P$. falciparum is sequestration of synchronously replicating parasites. The pattern of 6-day periodicity can be explained as the result of synchronicity during sequential rounds of replication. Young parasites present in the circulation at one time-point will have gone through 1.5 replication cycles at the next timepoint, 3 days later. As mature forms they will be cytoadhering in the body tissue and will not be sampled. At the subsequent time-point, they will once again be ring-stage parasites in the peripheral blood.

The 3-27 day time-intervals over which the periodicity was most prominent could be interpreted as the continuance of single, synchronous infections. Episodes of infection of this duration were observed within a single individual. Equally valid is the possibility that multiple asymptomatic $P$. falciparum infections in a single individual have a tendency to synchronize with each other.

This may be the case if synchronicity results from the effects of immune responses targeted against shared determinants or immunity which is genotypeindependent in nature. It must be noted that although statistical analysis indicates a tendency for periodicity, not all $P$. falciparum infections in all children showed this pattern. Children with the highest $P$. falciparum prevalence were smear positive 
at consecutive time-points. This could be a result of non-synchronous populations, multiple broods of a single population or multiple populations that are replicating out of phase.

The lack of periodicity in $P$. vivax and $P$. malariae conditional probability data was expected because they are non-sequestering species. Synchrony of the replicating cycle may occur in asymptomatic $P$. vivax and $P$. malariae populations. Determination of the different parasite stages present would be required to demonstrate this, as all stages of these species circulate in the peripheral blood. If such data were available for this data set, periodicity could not be detected in $P$. malariae, as the replication cycle of this species is equal in length to the sampling interval.

Periodic fluctuation in $P$. falciparum parasite density has been observed previously in asymptomatic residents of an endemic region sampled daily (Farnert et al. 1997) and has been extensively studied in symptomatic patients (White, Chapman \& Watt, 1992). Synchronicity of single populations has long been known and results in the periodic fever that is characteristic of malarial disease. It has been suggested that host immune responses and fever in particular can promote synchrony (Kwiatkowski, 1989; Kwiatkowski \& Greenwood, 1989). Fever is unlikely to be a factor in synchronization of asymptomatic infections in this population as the frequency of fever (2-3 febrile episodes per year in children of this age (Cox et al. 1994)) is unlikely to be sufficient to maintain synchrony. Alignment of the parasite replication cycle with the human circadian rhythm has been suggested as a mechanism underlying synchronicity (Hawking, Worms \& Gammage, 1968). This possibility is further investigated in a subset of these children in which $P$. falciparum genotype dynamics were determined (Bruce et al. $2000 b)$.

The comparison of daily and period prevalence confirms the underestimate that point prevalence makes in the malaria burden in a human population. Patent infection was detected in all but 1 individual over 1 month. Similar findings have been made in other endemic regions, Nigeria (Bruce-Chwatt, 1963) and Senegal (Trape et al. 1994) but the relationship of these results to the duration of infection in different age groups has not previously been investigated. The increasing difference between point and period prevalence with age suggests changes in the dynamics of infection with age. A decrease in the number of infections that result in detectable parasitaemia or a reduction in the duration of patent episodes of infection (or a combination of both of these factors) would be manifest in this way.

Classically, duration of malaria infection is taken to mean the total length of the period of infection with any Plasmodium species. This is calculated from models that estimate transition rates between para- sitaemic and non-parasitaemic states from pairs of samples taken weeks apart (Bekessy, Molineaux \& Storey, 1976; Kitua et al. 1996). However, multiple peaks of parasitaemia are known to arise from a single infection (Brown \& Brown, 1965; Handunnetti, Mendis \& David, 1987) and parasitaemia often falls to undetectable levels between peaks. These dynamics are thought to be a result of antigenic switching of parasite encoded, infected erythrocyte surface molecules (Gilks, Walliker \& Newbold, 1990; Baruch et al. 1995; Al-Khedery, Barnwell \& Galinski, 1999). Two-state models do not account for multiple peaks of infection, breakdown under large numbers of transitions and are of little value in areas with a high incidence of superinfection.

Our measurements are not of total duration of infection but reveal the duration of individual bursts of patent parasitaemia caused by each species. The dynamics of episodes of infection relate to the underlying rates of parasite population growth and clearance of parasites by the host immune system. Sequential bursts of parasitaemia of a single species in a single individual, could be a result of independently inoculated, distinct populations of parasites, or could result from recrudescence of a single population undergoing antigenic variation. Primary infections could not be distinguished from recrudescent ones as transmission was on-going throughout the study. The range of the duration of episodes represents a combination of these types of infections.

Immunity that clears parasites is known to be species-specific (Taliaferro, 1939; Jeffery, 1966; Jarra \& Brown, 1985) and the species-specific duration of infection observed here is consistent with this. The lower density attained by $P$. vivax and $P$. malariae infections may result in underestimation of duration as these species approach the microscopy threshold more frequently. This may contribute to the trend of shorter duration of infection compared to $P$. falciparum. However, the observation that the longer duration of $P$. falciparum episodes compared to $P$. vivax or $P$. malariae is maintained across all age groups, where there are varying density ranges for each species, points to the possibility of a real difference in the efficiency of acquired immunity against these species.

Such a difference is consistent with the quicker acquisition of immunity to $P$. vivax compared to $P$. falciparum (Ciuca, Ballif \& Chelarescu-Vieru, 1934) and may underlie the younger age of peak of $P$. vivax incidence compared to $P$. falciparum in areas where both of these species are present (Balfour, 1935; Earle et al. 1939; Maitland et al. 1996). The reduction in duration of episodes with age across all parasite species, along with the reduction in parasite density of $P$. falciparum and $P$. vivax in adults compared to children suggests that in older people 
immune responses are either triggered earlier in the growth of parasite populations or are more efficient at clearing parasites once triggered.

Age-specific differences in the duration of episodes of infection can partly explain the decline in age-prevalence curves in older age groups. The largest difference in duration was seen between the 4-year-olds and the 5-14 year age group. Peak $P$. falciparum point prevalence in this data set was in the 4-year-olds but in large surveys in this region is in the 5 to 9 -year-old age group (Cattani et al. 1986). Thus, the decline in prevalence which occurs in older age groups cannot be fully explained by differences in duration of episodes of infection, which are greatest before prevalence peaks.

Interactions between different infecting species and genotypes of parasite have been shown to alter Plasmodium dynamics in some children in this study (Bruce et al. 2000a). Density-dependent immune regulation that acts against all species is thought to underlie these interactions. This regulation is unlikely to alter the relative duration of each episode as it acts only to control parasite density around a threshold and is not responsible for ultimate parasite clearance.

Data from blood smears is useful in making general comparisons between species and across age groups. However, interpretation of these estimates is always limited by the uncertainty of the genetic identity of parasites of each infection in populations undergoing constant super-infection. As microscopy cannot distinguish individual infections below the species level episodes may be the result of infection with more than one genotype of any given species. This limitation has been addressed by determining the dynamics of individual genotypes of $P$. falciparum and $P$. vivax parasites from a subset of children described here. The results of this analysis are presented in the following paper (Bruce et al. $2000 b)$.

We would like to thank the people of Gonoa for their longstanding co-operation and for their tolerance throughout the study period. Our thanks also to the staff of the Papua New Guinea Institute of Medical Research at Madang for their assistance, and especially to the members of the field team who assisted with sample collection. This work is dedicated to the memory of Helena Vrbova and Nicky Gibson, whose contribution to malaria research in PNG was substantial. Field work was funded by a grant from The European Commission, STD 3, to D.W., K.P.D. and M.P.A. inc. K. D. and C.A.D. are funded by The Wellcome Trust. M. B. was funded by a studentship from The British Medical Research Council and by The Wellcome Trust.

\section{REFERENCES}

AL-KHEDERY, B., BARNWELL, J. W. \& GALINSKI, M. R. (1999). Antigenic variation in malaria: A $3^{\prime}$ genomic alteration associated with the expression of a $P$. knowlesi variant antigen. Molecular Cell 3, 131-141.
BALfour, M. C. (1935). Malaria studies in Greece. American Fournal of Tropical Medicine 15, 301-330.

BARUCH, D. I., PASlOSKE, B. L., SINGH, H. B., BI, X., MA, X. C., FELdMAN, M., TARASChi, T. F. \& HOWARD, R. J. (1995). Cloning the Plasmodium falciparum gene encoding PfEMP1, a malarial variant antigen and adherence receptor on the surface of parasitized human erythrocytes. Cell 82, 77-87.

BeKessy, A., Molineaux, L. \& STOREy, J. (1976). Estimation of incidence and recovery rates of Plasmodium falciparum parasitaemia from longitudinal data. Bulletin of the World Health Organization 54, 685-691.

BROWN, K. N. \& BROWN, I. N. (1965). Immunity to malaria: antigenic variation in chronic infections of Plasmodium knowlesi. Nature, London 208, 1286-1288.

BRUCE, M. C., DONNELly, C., ALPERS, M. P., GALinsKi, M. R., BARWELL, J. W., WALlikeR, D. \& DAY, K. P. $(2000 a)$. Cross-species interactions between malaria parasites in humans. Science 287, 845-848.

BRUCE, M. C., GALINSKI, M., BARNWELl, J. W., DONNELLY, C. A., Walmsley, M., Alpers, M., Walliker, D. \& DAY, K. P. $(2000 b)$. Genetic diversity and dynamics of $P$. falciparum and $P$. vivax populations in multiply infected children with asymptomatic malaria infections in Papua New Guinea. Parasitology 121, 257-272.

BRUCE-CHWATT, L. J. (1963). The longitudinal survey of natural malaria infection in a group of West African adults. West African Medical Fournal August, 141-217.

Burkot, T. R., Graves, P. M., CAtTani, J. A., Wirtz, R. A. \& GiBson, F. D. (1987). The efficiency of sporozoite transmission in the human malarias, Plasmodium falciparum and P. vivax. Bulletin of the World Health Organization 65, 375-380.

BURKot, T. R., GRAVES, P. M., PARU, R., WIRTZ, R. A. \& HEYWOOD, P. F. (1988). Human malaria transmission studies in the Anopheles punctulatus complex in Papua New Guinea: sporozoite rates, inoculation rates and sporozoite densities. American Fournal of Tropical Medicine and Hygiene 39, 135-144.

CAttani, J. A., tulloch, J. L., VRbova, H., JOlley, D., GIBSON, F. D., MOIR, J. S., HEYWOOD, P. F., ALPERS, M. P., STevenson, A. \& Clancy, R. (1986). The epidemiology of malaria in a population surrounding Madang, Papua New Guinea. American Fournal of Tropical Medicine and Hygiene 35, 3-15.

CiUCA, M., BALlif, L. \& CHELARESCU-VIERU, M. (1934). Immunity in malaria. Transactions of the Royal Society of Tropical Medicine and Hygiene 27, 619-622. COX, M. J., KuM, D., TAvul, L., NARARA, A., RAiko, A., AlPers, M., MEDley, G. \& DAY, K. P. (1994). Dynamics of malaria parasitaemia associated with febrile illness in children from a rural area of Madang, Papua New Guinea. Transactions of the Royal Society of Tropical Medicine and Hygiene 88, 191-197.

EARle, W. C., PEREZ, M., DEL RiO, J. \& ARZOla, C. (1939). Observations on the course of naturally acquired malaria in Puerto Rico. Puerto Rican Fournal of Public Health and Tropical Medicine 14, 391-406.

FÄrNERT, A., SNOUNOU, G., ROOTH, I. \& BJÖRKMAN, A. (1997). Daily dynamics of Plasmodium falciparum subpopulations in asymptomatic children in a 
holoendemic area. American Fournal of Tropical Medicine and Hygiene 56, 538-547.

GILKS, C. F., WALlikeR, D. \& NEWBold, C. I. (1990).

Relationships between sequestration, antigenic variation and chronic parasitism in Plasmodium chabaudi chabaudi - a rodent malaria model. Parasite Immunology 12, 45-64.

GILLES, H. M. (1993). Epidemiology of malaria. In BruceChwatt's Essential Malariology (ed. Gilles, H. M. \& Warrell, D. A.), pp. 124-164. Edward Arnold, , London.

GREENWOOD, B. M. (1987). Asymptomatic malaria infections - do they matter? Parasitology Today $\mathbf{3}$, 206-213.

GREENWOOD, B. M., BRADLEY, A. K., GREENWOOD, A. M., BYaSS, P., JAMMEH, L., MARSh, K., TUlloch, S., OLDFIELD, F. S. T. \& HAYES, R. (1987). Mortality and morbidity from malaria among children in a rural area of The Gambia, West Africa. Transactions of the Royal Society of Tropical Medicine and Hygiene 81, 478-486.

HANDUNNETTI, S. M., MENDIS, K. N. \& DAVID, P. H. (1987). Antigenic variation of cloned Plasmodium fragile in its natural host Macaca sinica: sequential appearance of successive variant antigenic types. Fournal of Experimental Medicine 165, 1269-1283.

HAWKing, F., WORMS, M. J. \& GAMMAGE, K. (1968). 24and 48-hour cycles of malaria parasites in the blood; their purpose, production and control. Transactions of the Royal Society of Tropical Medicine and Hygiene 62, 731-760.

hill, R. B., Cambournac, F. J. C. \& Simoes, M. P. (1943). Observations on the course of malaria in children in an endemic region. American Fournal of Tropical Medicine 23, 147-162.

JARRA, W. \& BROWN, K. N. (1985). Protective immunity to malaria: studies with cloned lines of Plasmodium chabaudi and P. bergei in CBA/Ca mice. I. The effectiveness and inter- and intra-species specificity of immunity induced by infection. Parasite Immunology 7, 595-606.

JEFFERY, G. M. (1966). Epidemiological significance of repeated infections with homologous and heterologous strains and species of Plasmodium. Bulletin of the World Health Organization 35, 873-882.

KitUa, A. Y., SMith, T., ALONSO, P. L., MASANJa, H., URASSA, H., MENENDEZ, C., KIMARIO, J. \& TANNER, M. (1996). Plasmodium falciparum malaria in the first year of life in an area of intense and perennial transmission. Tropical Medicine and International Health 1, 475-484.
KWiatKowski, D. (1989). Febrile temperatures can synchronise the growth of Plasmodium falciparum in vitro. Fournal of Experimental Medicine 169, 357-361.

KWIATKOWSKI, D. \& GREENWOOD, B. M. (1989). Why is malaria fever periodic? A hypothesis. Parasitology Today 5, 264-266.

MAITLAND, K., WILliaMs, T. N., BENNETT, S., NEWBOLD, C. I., PETO, T. E. A., Viji, J., TIMOThy, R., CleGG, J. B., Weatherall, D. J. \& Bowden, D. K. (1996). The interaction between $P$. falciparum and $P$. vivax in children on Espiritu Santo, Vanuatu, South West Pacific. Transactions of the Royal Society of Tropical Medicine and Hygiene 90, 614-620.

MILLER, M. J. (1958). Observations on the natural history of malaria in the semi-resistant West African. Transactions of the Royal Society of Tropical Medicine and Hygiene 52, 152-168.

Molineaux, L. (1988). The epidemiology of human malaria as an explanation of its distribution, including some implications for its control. In Malaria: Principles and Practice of Malariology, Vol. 2 (ed. Wernsdorfer, W. H. \& McGregor, I.), pp. 913-998. Churchill Livingstone, London.

ROGIER, C., COMMENGES, D. \& TRAPE, J. F. (1996). Evidence for an age-dependent pyrogenic threshold of Plasmodium falciparum parasitemia in highly endemic populations. American Fournal of Tropical Medicine and Hygiene 54, 613-619.

SMith, T., HURT, N., TEUSCHER, T. \& TANNER, M. (1995). Is fever a good sign for clinical malaria in surveys of endemic communities? American Fournal of Tropical Medicine and Hygiene 52, 306-310.

TALIAfERro, W. H. (1939). Immunity to the malaria infections. In Malariology (ed. Boyd, M. F.), pp. 935-965. W. B. Saunders \& Co., Philadelphia.

TRAPE, J. F., ROGIER, C., KONATE, L., DiAgne, N., Bouganali, H., CANQUe, B., Legros, F., Badji, A., NDiAye, G., NDIAYE, P., BRAhimi, K., FAYE, O., DRUilhe, P. \& PEREIRA DA SIlva, L. (1994). The Dielmo project: a longitudinal study of natural malaria infection and the mechanisms of protective immunity in a community living in a holoendemic area of Senegal. American Fournal of Tropical Medicine and Hygiene 51, 123-137.

white, N. J., Chapman, D. \& Watt, G. (1992). The effects of multiplication and synchronicity on the vascular distribution of parasites in falciparum malaria.

Transactions of the Royal Society of Tropical Medicine and Hygiene 86, 590-597. 